\title{
Positron Emission Tomography in Detection of Metastatic Leiomyosarcoma in a Postoperative Patient: A Case Report
} \author{
David B. Engle, MD., ${ }^{1}$ Linda M. Smiley, MD., ${ }^{2}$ Scott L. Baum, MD., ${ }^{3}$ Greg P.
Wellman, MD. ${ }^{4}$
}

Key Words: positron emission tomography, leiomyosarcoma, metastasis, diagnosis, postoperative

${ }^{1}$ The Department of Obstetrics and Gynecology, The Carver College of Medicine, The University of lowa, lowa City, Iowa, USA

${ }^{2}$ West Clinic, Division of Gynecologic Oncology, Memphis, TN, USA

${ }^{3}$ West Clinic, Division of Radiology, Memphis, TN, USA

4 University of Tennessee at Memphis, Department of Pathology, Memphis, TN, USA

\section{Introduction}

In leiomyosarcoma (LMS) abnormal vaginal bleeding is the most common reported symptom in patients $(56 \%)$, followed by pelvic mass (54\%), and pain $(22 \%) .{ }^{1}$ LMS is often hard to diagnosis on a uterine biopsy because it does not originate in the endometrium and may not invade into the cavity. In reality LMS is only detectable by endometrial biopsy in $33 \%$ of cases. $^{2}$ Non-specific symptoms as well as difficulty in diagnosis being made by biopsy, means that many LMS tumors are often mistaken for fibroids preoperatively.

If LMS is known preoperatively then a total abdominal hysterectomy with bilateral salpingo-oopherectomy (TAH/BSO) and evaluation of the abdominal and pelvic cavities can by undertaken. While routine pelvic and paraaortic lymphadenectomies are controversial in LMS, it may be required in certain circumstances. Unfortunately, a simple hysterectomy or even myomectomy is often performed for what is believed to be a benign process.

Please cite this paper as: Engle DB, Smiley LM, Baum SL, Wellman GP. Towards a Positron Emission Tomography in detection of metastatic leiomyosarcoma in a postoperative patient: a case report. Proc Obstet Gynecol. 2011 July;2(1):Article 14[5 p]. Available from: http://ir.uiowa.edu/pog/. Free full text article.

Corresponding author: David B. Engle, MD. Department of Obstetrics and Gynecology, University of Iowa Carver College of Medicine, 4630 JCP, 200 Hawkins Drive, Iowa City, IA 52242. Telephone (319)356-2015.Dbengle3@yahoo.com

This is an Open Access article distributed under the terms of the Creative Commons Attribution 3.0 Unported License (http://creativecommons.org/licenses/by/3.0), which permits unrestricted use, distribution, and reproduction in any medium, provided the original work is properly cited. 
Leiomyosarcoma is a rare tumor that accounts for only $1 \%$ of all uterine malignancies. ${ }^{1}$ In the past it was believed to be the most common uterine sarcoma; however a more recent report shows that it is second behind mixed mesodermal stromal sarcoma which accounts for $66 \%$ of uterine sarcomas. ${ }^{3}$ LMS can arise from either the smooth muscle cells of the myometrium or from within a leiomyomata (fibroid) itself . ${ }^{3}$

LMS often spreads by the hematogenous route with lung or pleural involvement as one of the most frequent metastatic sites. Five year overall survival rates for LMS by stage are: stage I $=60 \%$; stage II $=35 \%$; stage III $26 \%$; and stage IV $13 \%{ }^{4}$

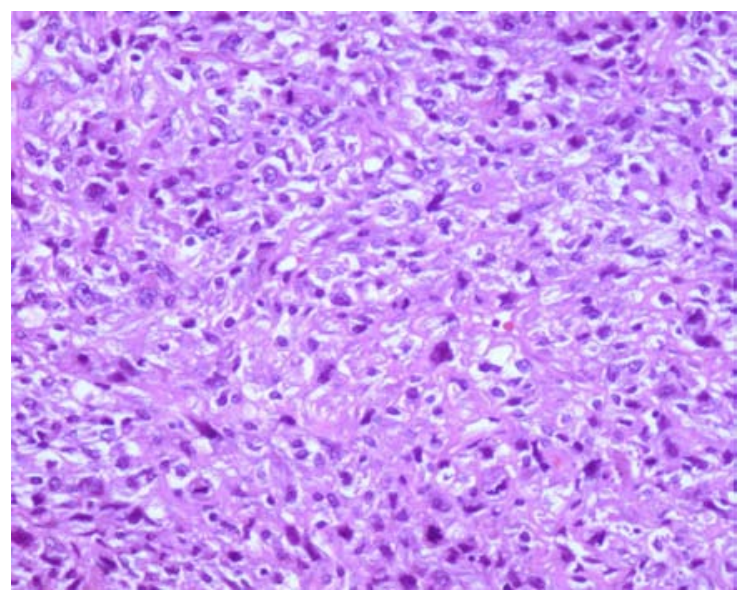

Figure 1

Histologically, the uterus displays hypercellularity and marked nuclear atypia with several mitotic figures, consistent with the diagnosis of leiomyosarcoma. (Hematoxylin and Eosin, original magnification: 200X)

\section{Case}

A 45-year-old G2, P2 presented to her primary Ob/Gyn for a 2-month history of worsening dysfunctional uterine bleeding. An ultrasound was performed and it was felt that this patient had a $7 \mathrm{~cm}$ fibroid (leiomyomata). The patient subsequently underwent total abdominal hysterectomy with bilateral salpingoopherectomy. Final pathology (fig. 1) showed a LMS with greater than 15 mitotic figures per 10 high power fields (>15mitosis/10HPF). Areas of coagulative necrosis were also noted; however, there was no vascular space involvement. Further tests confirmed this tumor had both estrogen and progesterone receptors.
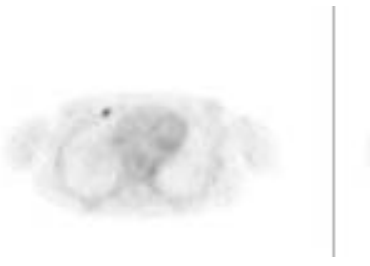

Figure 2

FDG-PET scan of chest, with suspicious lesion in black

This patient's care was subsequently transferred to a Gynecologic Oncologist at The West Clinic in Memphis, Tennessee. Postoperatively the patient did very well, and a computed tomography (CT) scan of the patient's chest, abdomen, and pelvis were obtained. On chest CT three nodules were noted. Two of these appeared to be benign granulomas, but the third nodule had an indeterminate appearance. A positron emission tomography scan (PET) using [18F]- 
fluorodeoxyglucose (FDG) was performed (fig 2). PET scan revealed a hypermetabolic lesion with a standardized uptake value (SUV) of 4.1. Subsequently a CT guided percutaneous needle biopsy was performed on the right pulmonary nodule (fig. 3). The lung biopsy was examined and compared to the patient's original hysterectomy slides, and metastatic LMS was confirmed (fig. 4).

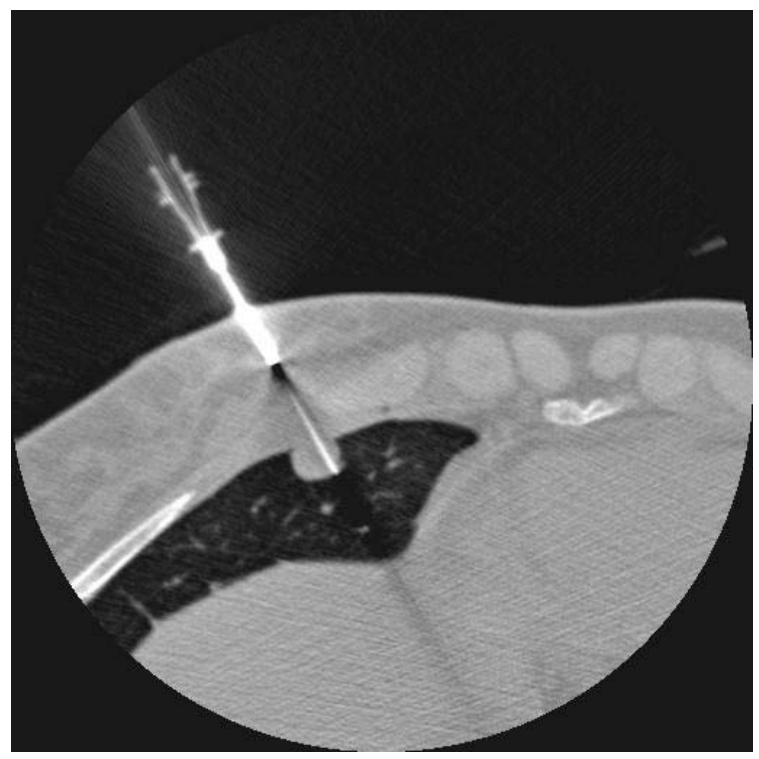

Figure 3

CT of chest with suspicious $9 \mathrm{~mm}$ nodule during biopsy

A small pneumothorax was noted after the biopsy procedure. The pneumothorax resolved spontaneously with time under observation. The patient underwent chemotherapy using a sarcoma protocol with Adriamycin and Ifosfamide. She was re-assessed after 2-cyles for consideration of ablation or resection of the lung nodule; however, the lesion decreased in size from $9 \mathrm{~mm}$ to less than $3 \mathrm{~mm}$ and was not felt to be amenable to resection. The patient completed 6 cycles of the above chemotherapy with resolution of the lung lesion. The patient is currently under observation.

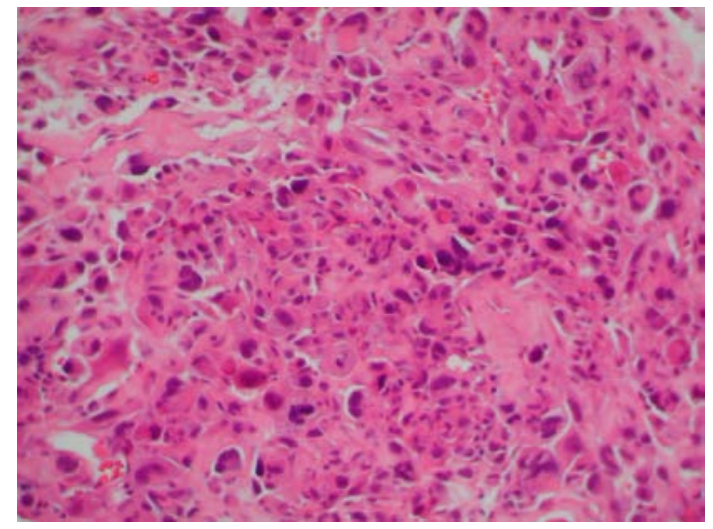

Figure 4

The bizarre cellular atypia and brisk mitotic rate of the primary lesion are recapitulated in this biopsy from the lung metastases. (Hematoxylin and Eosin, original magnification: 200X)

\section{DISCUSSION}

Positron Emission Tomography (PET) is now commonplace in the realm of oncology, and Fludeoxyglucose (FDG) has become a common tracer. FGD is a glucose analogue that is readily taken up by cells using the glucose transporter, in particular by tumor cells. ${ }^{6}$ Tumor cells have a higher metabolic rate then normal cells causing greater FDG uptake. ${ }^{7}$ FDG cannot be metabolized like glucose, and therefore is "trapped" within the cell. These areas of increased FDG uptake, or increased SUV, are used to show a 3 dimensional image of the body with areas of concern highlighted. 
FDG-PET has been used successfully in other gynecological malignancies. Hubner et al. in his study of ovarian cancer and FDGPET found PET had $93 \%$ sensitivity and $82 \%$ specificity in primary ovarian tumors. He also showed that when CT and FDG-PET were combined they had a $95 \%$ positive predictive value and a $100 \%$ negative predictive value. ${ }^{8}$

FDG-PET has also been used successfully in cervical cancer. Sugawara showed that FDG-PET correctly identified 16 of 21 known cervical cancers. These results were improved when post bladder void scans was done and correctly identified 11 of 11 patients. ${ }^{9}$ FDG is excreted in the urine, and therefore the bladder will concentrate the tracer making pelvic images difficult to evaluate.

FDG-PET has already been used successfully in the preoperative assessment of uterine sarcomas. A study done in Osaka, Japan looked at the preoperative scans of 5 patients with uterine sarcoma. Of these 5 patients with pathology confirmed sarcoma all 5 patients had positive (SUV >2.5) FDG-PET scans. 3 of these reported 5 patients had LMS, 1 had carcinosarcoma, and the last one had endometrial stromal sarcoma. ${ }^{10}$

Another preoperative study done by Nagamatsu et al. showed an average SUV of 4.2 in their group of 4 LMS. $^{11}$ They showed that SUV uptake in endometrial carcinoma, carcinosarcoma, and LMS had a higher SUV than benign leiomyomas. They further showed that the combination of FDG-PET and serum $\mathrm{LDH}$ had improved sensitivity and specificity than FDGPET alone for detecting LMS.

Kao et al. looked at the use of FDGPET and CT combination to detect recurrent disease in LMS. ${ }^{12}$ Among 11 patients they found the combination scan had near perfect accuracy. However, 2 patients had recurrence only detected by the CT portion and not by PET.

To our knowledge this is the only reported case of FDG-PET being used in the postoperative evaluation of a patient with LMS and a suspicious lung mass. Our case shows there may be a place for PET scans in the post-op surveillance of LMS. This method would be ideally suited, considering the metastatic spread pattern of LMS. Unfortunately due to the rarity of this disease a prospective evaluation of this modality would not be practical.

\section{References}

1. Giuntoli RL 2nd, Metzinger DS, DiMarco CS, Cha SS, Sloan JA, Keeney GL, Gostout BS. Retrospective review of 208 patients with leiomyosarcoma of the uterus: prognostic indicators, surgical management, and adjuvant therapy. Gynecol Oncol. 2003 Jun;89(3):4609.

2. Schwartz LB, Diamond MP, Schwartz PE. Leiomyosarcomas: clinical presentation. Am J Obstet Gynecol. 1993 Jan;168(1 Pt 1):1803.

3. DiSaia PJ, Creasman WT. Sarcoma of the Uterus. In: DiSaia 
PJ, Creasman WT, editors. Clinical Gynecologic Oncology. 6th ed. St. Louis: Mosby; 2002. p 173-84.

4. Kosary CL. Cancer of the corpus uteri. In: National Cancer Institute. SEER Survival Monograph. Bethesda, MD: NCl, 2008.

5. Bomanji JB, Costa DC, Ell PJ. Clinical role of positron emission tomography in oncology. Lancet Oncol. 2001 Mar;2(3):157-64.

6. Horowitz NS, Dehdashti F, Herzog TJ, Rader JS, Powell MA, Gibb RK, Grigsby PW, Siegel BA, Mutch DG. Prospective evaluation of FDG-PET for detecting pelvic and para-aortic lymph node metastasis in uterine corpus cancer. Gynecol Oncol. 2004 Dec;95(3):546-51.

7. Hubner KF, McDonald TW, Niethammer JG, Smith GT, Gould $\mathrm{HR}$, Buonocore E. Assessment of primary and metastatic ovarian cancer by positron emission tomography (PET) using 2[18F]deoxyglucose (2-[18F]FDG). Gynecol Oncol. 1993 Nov;51(2):197-204.
8. Sugawara $Y$, Eisbruch A, Kosuda S, Recker BE, Kison PV, Wahl RL. Evaluation of FDG PET in patients with cervical cancer. J Nucl Med. 1999 Jul;40(7):1125-31.

9. Umesaki N, Tanaka T, Miyama M, Kawamura N, Ogita S, Kawabe J, Okamura T, Koyama K, Ochi $\mathrm{H}$. Positron emission tomography with (18)F-fluorodeoxyglucose of uterine sarcoma: a comparison with magnetic resonance imaging and power Doppler imaging. Gynecol Oncol. 2001 Mar;80(3):372-7.

10. Nagamatsu A, Umesaki N, Li L, Tanaka T. Use of 18Ffluorodeoxyglucose positron emission tomography for diagnosis of uterine sarcomas. Oncol Rep. 2010 Apr;23(4):1069-76.

11. Kao $\mathrm{YH}$, Saad $U$, Tan AE, Magsombol BM, Padhy AK. Fluorine-18-fluorodeoxyglucose

12. PET/CT for the evaluation of suspected recurrent uterine leiomyosarcomas. Acta Radiol. 2011 May 1;52(4):463-6. 